\title{
Evaluación del uso de conservantes: sorbato de potasio, acetatos y diacetatos y mezcla de sorbato de potasio y ácido cítrico en la vida útil del producto comercial "sangrecita de pollo"
}

Evaluation of the use of preservatives: potassium sorbate, acetates and diacetates and mixture of potassium sorbate and citric acid in the useful life of the commercial product "sangrecita de pollo"

Recibido: diciembre 12 del 2018 | Revisado: enero 17 del 2019 | Aceptado: febrero 18 del 2019

\author{
Giovanna Castañeda SaAvedra*i \\ Silvia Ara Rojas ${ }^{\mathrm{I}-2}$ \\ Rosa Aguirre Medrano ${ }^{\mathrm{I}-3}$ \\ Edy Barnett Mendoza ${ }^{\mathrm{I}-4}$ \\ Ana María Bernabel Liza ${ }^{\mathrm{I}-5}$ \\ Luis Celi SaAvedra ${ }^{\mathrm{I}-6}$ \\ Mónica Ramos Escudero ${ }^{\mathrm{I}-7}$
}

* Autor correspondiente: M.Sc.Giovanna Castañeda

1 Facultad de Ingeniería y Arquitectura, Escuela de Ingeniería Alimentaria, Universidad de San Martín de Porres, Lima - Perú

mcastanedas@usmp.pe

2 sarar@usmp.pe

3 raguirrem@usmp.pe

4 ebarnettm@usmp.pe

5 abernabell@usmp.pe

6 lceli@usmp,pe

7 mramose@usmpe.pe

\section{RESUMEN}

El objetivo del proyecto de investigación fue evaluar la acción antimicrobiana de tres tipos de presevantes: ácido ascórbico, acetato-diacetato y la combinación de ácido cítrico -sorbato de potasio en la vida útil del plato típico peruano sangrecita elaborado con sangre de pollo, reconocido en nuestro medio por su potencial de hierro que puede complementar la oferta de alimentos promovidos por el Ministerio de Salud para combatir la desnutrición y la anemia en poblaciones vulnerables. En la primera etapa de ejecución de la investigación se procedió a desarrollar la formulación base del producto comercial sangrecita de pollo, y en base a esta la aplicación de los tres preservantes alimenticios considerados en la investigación procediéndose a su envasado y sellado al vacío. Para la determinación de vida útil se realizaron análisis fisicoquímicos, microbiológicos y de evaluación sensorial del producto comercial sangrecita de pollo envasado al vacío y almacenado a temperatura de refrigeración de $8^{\circ} \mathrm{C}$ por espacio de 20 días. De las evaluaciones realizadas, se determinó que de los tres tipos de preservantes alimenticios utilizados, el acetato más diacetato fue el que mantuvo en el producto elaborado recuentos de microrganismos menores hasta los 20 días, donde los recuentos si bien superaban el límite inferior (establecido por la Norma de $5 \times 10^{4}$ $\mathrm{UFC} / \mathrm{g}$ ), se mantuvieron por debajo del límite superior establecido $\left(5 \times 10^{5} \mathrm{UFC} / \mathrm{g}\right)$ siendo aún aptos para su consumo.

Palabras clave: producto comercial sangrecita de pollo, preservantes alimenticios y vida útil

\section{Abstract \\ The objective of the research project was to evaluate the antimicrobial action of three types of preservatives:}

(C) Los autores. Este artículo es publicado por la Revista Campus de la Facultad de Ingeniería y Arquitectura de la Universidad de San Martín de Porres. Este artículo se distribuye en los términos de la Licencia Creative Commons Atribución No-comercial - Compartir-Igual 4.0 Internacional (https://creativecommons.org/licenses/ CC-BY), que permite el uso no comercial, distribución y reproducción en cualquier medio siempre que la obra original sea debidamente citada. Para uso comercial contactar a: revistacampus@usmp.pe. 
ascorbic acid, acetate-diacetate and the combination of citric acid with potassium sorbate in the useful life of the typical Peruvian dish "sangrecita de pollo" made with chicken blood, recognized in our environment for its potential iron content, which can be a nutritional supplement promoted by the Ministry of Health to combat malnutrition and anemia in vulnerable populations. In order to develop the commercial product "Sangrecita de pollo", in a first stage of the research was formulated, assessed and characterized the product and the application of the three preservatives established in the investigation, finally it was packed and vacuum sealed.For the determination of the useful life of the commercial product, physical-chemical, microbiological and sensory evaluation analyses were carried out after being stored for 20 days at $8^{\circ}$ Celsius of temperature. Of the evaluations carried out, it was determined that of the three types of preservatives used, the acetate-diacetate was the one that maintained in the elaborated product count of microorganisms' minor until the 20 days, although they exceeded the lower limit of count of Microorganisms (established by the $5 \times 10^{4}$ standard UFC/g) were kept below the established upper limit $\left(5 \times 10^{5} \mathrm{ufc} / \mathrm{g}\right)$ being still suitable for consumption.

Key words: Commercial product "Sangrecita de pollo", food preservatives, and useful life

\section{Introducción}

El Ministerio de Salud en el marco de su política de apoyo a poblaciones vulnerables ha priorizado revertir la deficiencia de hierro promoviendo la búsqueda de alternativas de desarrollo de productos alimenticios con elevados contenidos de hierro que tengan aceptación por parte de estas poblaciones. De acuerdo a la Organización Mundial de la Salud esta deficiencia se constituye en un problema de salud pública que se manifiesta por la insuficiente cantidad de glóbulos rojos en la sangre, lo cual está asociado con la mortalidad infantil, materna y el bajo peso al nacer. Al respecto, según esta entidad, la anemia se puede prevenir a bajo costo si se consumen alimentos ricos en hierro como la sangre de pollo, bazo bovino y otras vísceras que por desconocimiento se eliminan en los mercados.

De acuerdo a lo expuesto se considera una alternativa comercial relevante en nuestro país donde la situación de deficiencia de hierro es preocupante, difundir y promover el consumo del plato típico peruano sangrecita de pollo, toda vez que el insumo base para su elaboración es la sangre de pollo, fuente de hierro al contener en cada 100 gramos, $27,3 \mathrm{mg}$ del mineral, contando además con ventajas de ser de fácil obtención y bajo costo.

Se planteó desarrollar una investigación aplicada tendiente a estandarizar la formulación del plato típico sangrecita de pollo, determinando parámetros de proceso y envasado, así como evaluación de su vida útil en condiciones de almacenaje a temperaturas de refrigeración aplicando tres tipos de conservantes alimenticios, y su efecto en el tiempo de vida de este producto. Los conservantes seleccionados fueron: sorbato de potasio, mezcla de acetatos y diacetatos y el uso de mezcla de sorbato de potasio y el ácido cítrico (seleccionados por su acción antimicrobiana y por no inhibir la biodisponibilidad del hierro en el producto). 


\section{Materiales y métodos}

La investigación se llevó a cabo en los Laboratorios de Físico -Química y el Laboratorio de Biología y Microbiología de la Escuela Profesional de Ingeniería en Industrias Alimentarias de la Facultad de Ingeniería y Arquitectura de la Universidad de San Martín de Porres.
Teniendo en cuenta los objetivos planteados en la investigación se procedió en la primera fase de ejecución del proyecto a desarrollar la formulación base del producto comercial sangrecita de pollo, teniendo en cuenta los ingredientes utilizados y la modalidad de presentación en el mercado, para posteriormente proceder a la aplicación de los tres preservantes alimenticios seleccionados y evaluar su efecto.

\section{Diagrama de proceso de elaboración producto comercial sangrecita de pollo}

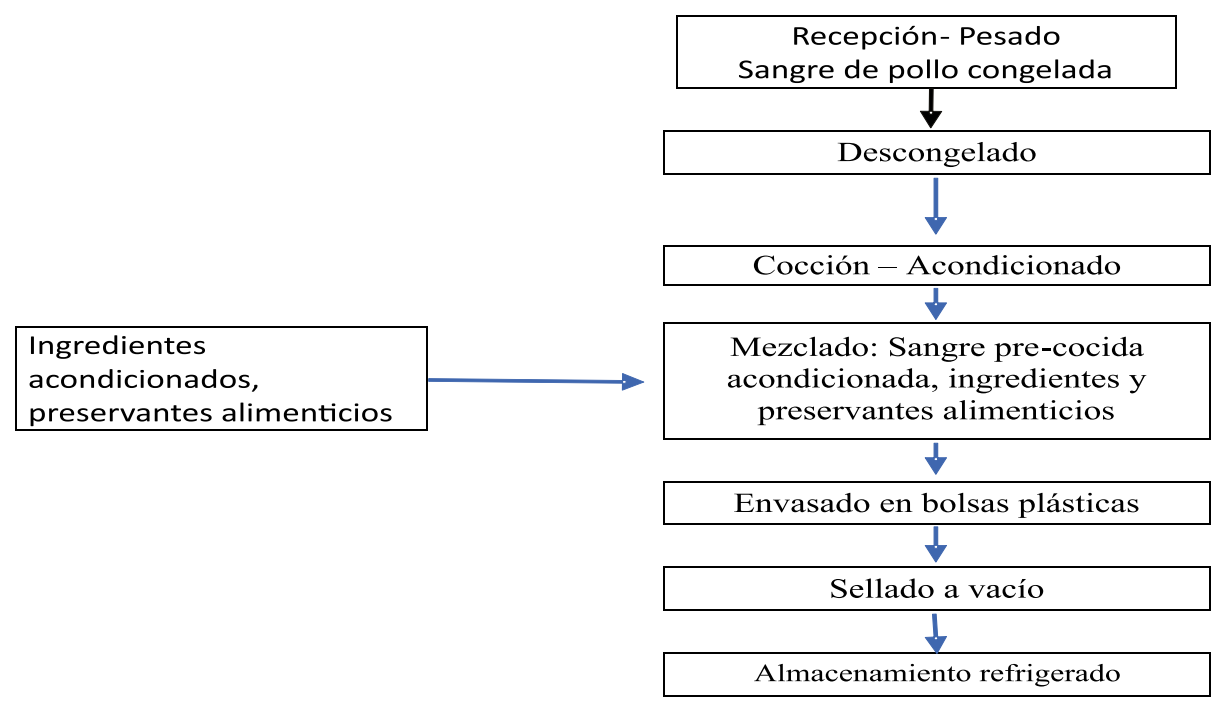

\section{Descripción del proceso}

- Selección y acondicionamiento de materia prima:

La materia prima base de la formulación, sangre de pollo, se seleccionó teniendo en cuenta las características físico-químicas y microbiológicas, según la norma técnica peruana (NTP). La temperatura de almacenamiento fue de $0^{\circ} \mathrm{C}-5^{\circ} \mathrm{C}$.

\section{- Pre cocción:}

La sangrecita se sometió a lavado y enjuagado, y posteriormente a tratamiento térmico a $80^{\circ} \mathrm{C}$ x 35 minutos, enfriándose luego a temperatura ambiente $\operatorname{los} 18^{\circ} \mathrm{C}$.

\section{- Picado / mezclado:}

El insumo base sangre de pollo pre cocida, así como las hortalizas conformantes de la formulación fueron picadas en porciones de $3 \mathrm{~mm}$ y se mezclaron con el resto de ingredientes de la formulación, incorporando posteriormente los preservantes alimenticios considerados en la investigación.

\section{- Envasado - sellado:}

Se procedió al envasado del producto sangrecita de pollo elaborado en bolsas de polietileno de alta densidad y selladas al vacío. El contenido de las bolsas fue de 500 gramos.

\section{- Enfriado-almacenaje}

La sangrecita de pollo envasada en bolsas de polietileno se almacenó a temperatura de refrigeración. 


\section{Caracterización del producto elaborado}

El producto comercial sangrecita de pollo, elaborado en base a la formulación desarrollada, fue caracterizado desde el punto de vista físico químico, microbiológico y de evaluación sensorial.

\section{Análisis Fisicoquímicos}

Análisis de proteínas. Método de Kjeldahl, (AOAC 976.06.), Análisis de humedad. Método ASTM D3302M ISO 589

Análisis de grasa por el método del soxhlet, (AOAC 920.39)

Análisis de cenizas por el método AOAC 923.03 a $550{ }^{\circ} \mathrm{C}$

Análisis de hierro por el método volumétrico corregido (Balta, 2007; Harris, 2015).

\section{Análisis microbiológico}

Los análisis microbiológicos se realizaron en el Laboratorio de Biología de la Facultad de Ingeniería y Arquitectura de la Universidad de San Martín de Porres de acuerdo a la Norma técnica de Salud No 071 de la Dirección General de Salud Ambiental del Ministerio de Salud.

Se realizó la evaluación de Aerobios mesófilos viables (AMV), recuento de Escherichia coli y ausencia en $25 \mathrm{~g}$ de Salmonella sp. El recuento de AMV se ejecutó siguiendo el protocolo establecido por la norma ISO 4833, el de E. coli y la determinación de Salmonella por la norma NF V08-052.

En Tabla 1 se presenta criterios de calidad microbiológica establecidos para este tipo de productos:

Tabla 1

Recuentos de microorganismos establecidos por la Norma Técnica

\begin{tabular}{lcccccc}
\hline Agente microbiano & Categoría & Clase & $\mathbf{n}$ & $\mathbf{C}$ & $\mathbf{m}$ & $\mathbf{M}$ \\
\hline Aerobios mesófilos viables & 3 & 3 & 5 & 1 & $5 \times 10^{4}$ & $5 \times 10^{5}$ \\
\hline Escherichia coli & 6 & 3 & 5 & 1 & 10 & $10^{2}$ \\
\hline Salmonella $\mathrm{sp}$ & 10 & 2 & 5 & 0 & Ausencia en $25 \mathrm{~g}$ & ----- \\
\hline
\end{tabular}

Se considera que el producto es apto para consumo el tiempo que mantenga los recuentos por debajo de los establecidos en la columna "M" y ausencia de Salmonella (Tabla 1).

\section{Análisis sensorial}

Se analizó mediante la prueba de Friedman, con una primera prueba de aceptación con un panel de 21 consumidores y una segunda prueba de calidad (prueba hedónica de cinco puntos en las que se calificó atributos de olor, sabor, textura y color) con un panel de 15 consumidores.

\section{Resultados}

\section{Análisis fisicoquímicos de la sangre de pollo}

En la Tabla 2 se presentan los resultados obtenidos de los análisis de la sangre de pollo fresca. 
EVALUACIÓN DEL USO DE CONSERVANTES: SORBATO DE POTASIO, ACETATOS Y DIACETATOS Y MEZCLA DE SORBATO DE POTASIO Y ÁCIDO CÍTRICO EN LA VIDA ÚTIL DEL PRODUCTO COMERCIAL “SANGRECITA DE POLLO”

Tabla 2

Resumen de análisis fisicoquimico de la sangre de pollo fresca

\begin{tabular}{cc}
\hline Determinaciones & Cantidad (\%) \\
\hline Proteínas & 20.47 \\
\hline Humedad & 74.0 \\
\hline Grasas & 0.12 \\
\hline Hierro & $469.61(\mathrm{mg} / \mathrm{kg})$ \\
\hline
\end{tabular}

Fuente: CERPER

\section{Formulación base del producto comer- cial sangrecita de pollo}

Se realizaron pruebas de evaluación sensorial a los productos elaborados, sangrecita de pollo, con las formulaciones recolectadas, lo que permitió establecer la formulación base para el desarrollo de la parte experimental del proyecto de investigación. Tabla 2.

Tabla 3

Formulación de sangrecita de pollo

\begin{tabular}{cc}
\hline Ingredientes & $\begin{array}{c}\text { Formulación } \\
\text { base } \%\end{array}$ \\
\hline Sangre & 56.88 \\
\hline Hierba buena & 9.58 \\
\hline Cebolla roja & 14.0 \\
\hline Cebolla china & 6.62 \\
\hline Perejil & 2.50 \\
\hline Ají amarillo & 2.36 \\
\hline Poro & 3.96 \\
\hline Proteína Aislada soya & 1.20 \\
\hline Carragel & 0.30 \\
\hline Sorbato/Ácido cítrico / & 0.30 \\
\hline lactatos & 2.0 \\
\hline Sal & 0.18 \\
\hline Ajos molido & 0.08 \\
\hline Comino & 0.08 \\
\hline Ajinomoto & 0.12 \\
\hline Orégano & 100.0 \\
\hline Total &
\end{tabular}

Fuente: Elaboración propia

\section{Caracterización de la sangrecita de po- llo elaborada}

En la Tabla 3 se presenta los resultados de los análisis físicos químicos realizados con el producto comercial sangrecita de pollo elaborado para el desarrollo de la fase experimental del proyecto.

Tabla 4

Resumen de análisis fisicoquimico del producto sangrecita de pollo

\begin{tabular}{cc}
\hline Determinaciones & Cantidad (\%) \\
\hline Proteínas & 14.47 \\
\hline Humedad & 78.3 \\
\hline Grasas & 0.24 \\
\hline \multirow{2}{*}{ Hierro } & $301.71(\mathrm{mg} /$ \\
& $\mathrm{kg})$ \\
\hline
\end{tabular}

Fuente: CERPER

Con la formulación desarrollada se procedió a la elaboración del producto comercial sangrecita de pollo con la adición de los preservantes alimenticios considerados en la investigación:

$\mathrm{SAC}=$ sorbato de potasio + ácido cítrico

$\mathrm{SK}=$ sorbato de potasio

$\mathrm{AD}=$ acetato + diacetato

\section{Análisis microbiológico}

El producto sangrecita de pollo elaborado fue evaluado microbiológicamente frente a los tres parámetros establecidos: aerobios mesófilos viables, Escherichia coli y ausencia de Salmonella sp en $25 \mathrm{~g}$ en los días 1, 15 y 20. Los recuentos obtenidos son mostrados en la Tabla 5. 
Tabla 5

Análisis microbiológico del producto sangrecita de pollo al día 1, 15 y 20 de su elaboración

\begin{tabular}{|c|c|c|c|c|c|c|}
\hline \multirow{2}{*}{ Parámetro } & \multicolumn{2}{|c|}{ Límite por g. } & \multirow{2}{*}{ Día } & \multicolumn{3}{|c|}{ Conservante } \\
\hline & $\mathbf{m}$ & M & & SAC & SK & AD \\
\hline \multirow{3}{*}{$\begin{array}{l}\text { Aerobios mesófilos viables } \\
\text { (UFC/g) }\end{array}$} & \multirow{3}{*}{$5 \times 10^{4}$} & \multirow{3}{*}{$5 \times 10^{5}$} & 1 & $9 \times 10^{2}$ & $2 \times 10^{3}$ & $2 \times 10^{3}$ \\
\hline & & & 15 & $2 \times 10^{4}$ & $3 \times 10^{4}$ & $2 \times 10^{4}$ \\
\hline & & & 20 & $2 \times 10^{5}$ & $3 \times 10^{5}$ & $1 \times 10^{5}$ \\
\hline \multirow{3}{*}{ Escherichia coli (UFC/g) } & \multirow{3}{*}{10} & \multirow{3}{*}{$10^{2}$} & 1 & 0 & 0 & 0 \\
\hline & & & 15 & 0 & 0 & 0 \\
\hline & & & 20 & 0 & 0 & 0 \\
\hline \multirow{3}{*}{ Salmonella $\mathrm{sp}$} & \multirow{3}{*}{$\begin{array}{l}\text { Ausencia } \\
\text { en 25g }\end{array}$} & \multirow{3}{*}{---- } & 1 & 0 & 0 & 0 \\
\hline & & & 15 & 0 & 0 & 0 \\
\hline & & & 20 & 0 & 0 & 0 \\
\hline
\end{tabular}

$\mathrm{SAC}=$ sorbato de potasio + ácido cítrico, $\mathrm{SK}$ = sorbato de potasio, $\mathrm{AD}$ = acetato + diacetato

\section{Análisis Sensorial}

Los resultados del primer análisis sensorial fueron evaluados estadísticamente mediante la Prueba de Análisis de Va- rianza de Friedman (Tabla 6). La evaluación de las características organolépticas se muestra en la Tabla 7 y su evaluación global en la Figura 1.

Tabla 6

Análisis de Varianza de Friedman de los valores de preferencia de cinco productos de "Sangrecita de pollo" con un $\alpha$ de 0.05 .

\begin{tabular}{lcccc}
\hline & Sabor & Textura & Color & Olor \\
\hline Chi-cuadrado & 7,223 & 6,340 & 6,601 & 7,068 \\
\hline Sig. asintótica & 0,125 & 0,045 & 0,159 & 0,032 \\
\hline
\end{tabular}

Tabla 7

Evaluación de las caracteristicas organolépticas de producto sangrecita de pollo

\section{Producto de "Sangrecita de pollo"}

\begin{tabular}{cccccc}
\hline Características & $\begin{array}{c}\text { Sorbato de } \\
\text { Potasio }\end{array}$ & Acetatos & $\begin{array}{c}\text { Ac. Cítrico+ Sorb. } \\
\text { de Potasio }\end{array}$ & Redondos & Wong \\
\hline Sabor & 3.9 & 4.0 & 4.3 & 3.5 & 3.9 \\
\hline Textura & 3.8 & 3.8 & 4.1 & 3.4 & 3.4 \\
\hline Color & 3.5 & 3.4 & 3.8 & 3.8 & 3.5 \\
\hline Olor & 3.8 & 3.9 & 4.3 & 3.7 & 3.5 \\
\hline Promedio & 3.7 & 3.8 & 4.1 & 3.6 & 3.6 \\
\hline
\end{tabular}


EVALUACIÓN DEL USO DE CONSERVANTES: SORbaTO DE POTASIO, ACETATOS Y DiACETATOS Y MEZCLA DE SORBATO DE POTASIO Y ÁCIDO CÍTRICO EN LA VIDA ÚTIL DEL PRODUCTO COMERCIAL “SANGRECITA DE POLLO”

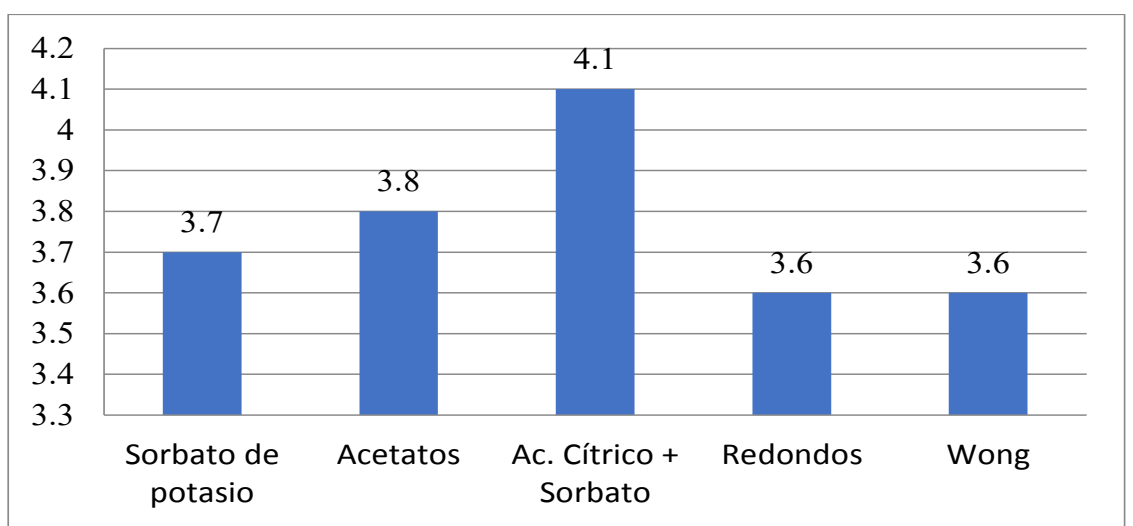

Figura 1. Evaluación global del producto sangrecita de pollo

Los resultados del segundo análisis sensorial fueron evaluados estadísticamente mediante la Prueba de Análisis de Varianza de Friedman (Tabla 8). La eva- luación de las características organolépticas es mostrada en la Tabla 9 y su evaluación global en la Figura 2.

Tabla 8

Análisis de Varianza de Friedman de los valores de preferencia de tres productos de "Sangrecita de pollo" con un $\alpha$ de 0.05 .

\begin{tabular}{lcccc}
\hline & Sabor & Textura & Color & Olor \\
\hline Chi-cuadrado & 4,962 & 4,000 & 6,048 & 1,250 \\
\hline Sig. asintótica & 0,084 & 0,135 & 0,049 & 0,535 \\
\hline
\end{tabular}

Tabla 9

Evaluación de las caracteristicas organolépticas de producto sangrecita de pollo

\begin{tabular}{lccc}
\hline \multirow{2}{*}{ Características } & \multicolumn{3}{c}{ Productos de "Sangrecita de pollo" } \\
\cline { 2 - 4 } & Sorbato de Potasio & Acetatos & $\begin{array}{c}\text { Ácido cítrico }+ \\
\text { Sorbato de Potasio }\end{array}$ \\
\hline Sabor & 3.5 & 3.5 & 4.0 \\
\hline Textura & 3.7 & 3.3 & 3.9 \\
\hline Olor & 3.5 & 3.6 & 3.9 \\
\hline Color & 3.5 & 3.3 & 3.9 \\
\hline Promedio & 3.5 & 3.4 & 3.9 \\
\hline
\end{tabular}

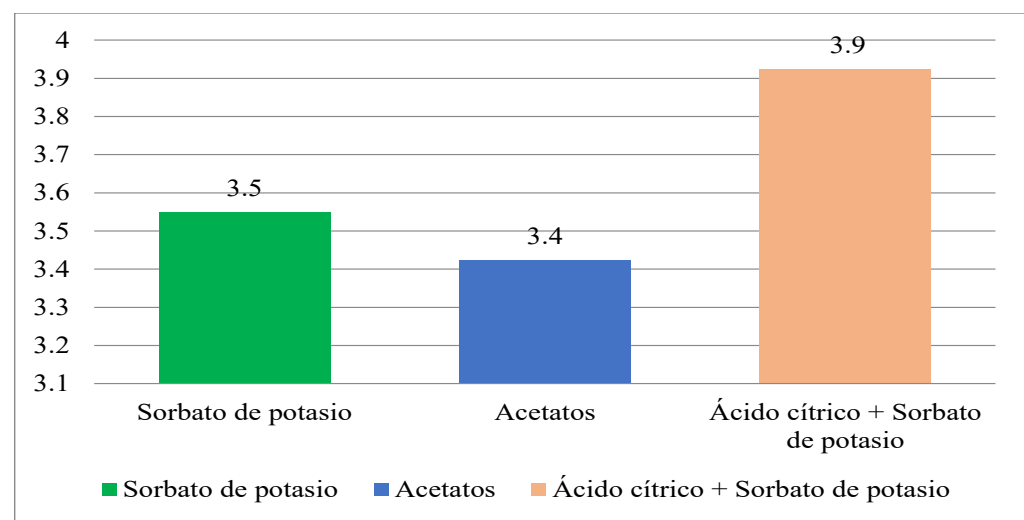

Figura 2. Evaluación global del producto sangrecita de pollo 


\section{Discusión}

De los resultados de los análisis fisicoquímicos realizados a la sangre de pollo fresca se aprecia su potencial valor alimenticio reportándose un nivel de $20.47 \%$ de proteína. Respecto al contenido de grasa, $0.12 \%$ que implica bajo aporte de grasa al cuerpo (65 calorías) es recomendable para personas con problemas de colesterol. El nivel de hierro que se reporta en la sangre de pollo de 469.61 $\mathrm{mg} / \mathrm{Kg}$ nos permite valorar la potencialidad de este insumo disponible y de bajo costo en la formulación de diferentes presentaciones de productos alimenticios, como es el caso de la sangrecita de pollo, en el marco de las campañas para disminuir el problema de la anemia en poblaciones vulnerables en nuestro país.

Los análisis fisicoquímicos realizados, a la sangrecita de pollo elaborada en el proyecto, reportan niveles de proteína de 14.475 , grasa $0.24 \%$ y contenidos de hierro de $301.71(\mathrm{mg} / \mathrm{Kg})$. Con respecto al hierro, el nivel encontrado en las muestra elaborada es mayor al reportado para otros alimentos como la morcilla $(190 \mathrm{mg} / \mathrm{kg})$, almejas $(240 \mathrm{mg} / \mathrm{kg})$ o las lentejas (33 mg/kg); (Galarza 2012). Es importante acotar que la sangrecita de pollo tiene hierro hemínico, no necesitando vitamina $\mathrm{C}$ o cítricos para una mejor absorción (Bueno 2015),

La Norma técnica de Salud No 071 no establece criterios microbiológicos para la sangrecita de pollo como alimento, pero sí lo hace para productos como la morcilla y el relleno que contienen sangre en su formulación. En esta norma (acápite 10.11) se establece como microorganismos determinantes de la inocuidad de estos alimentos a los aerobios mesófilos
(AMV), Escherichia coli, Staphylococcus aureus, Clostridium perfringens, Salmonella spp y Listeria monocitogenes. De los cuales $C$. perfringens y L. monocitogenes están presentes en componentes cárnicos de estos productos y $S$. áureus en la cubierta o tripa utilizada por efecto de contaminación por manipuladores al momento de realizar el embutido (Rengifo y col. 2010; Ruiz, 2009).

Considerando estos aspectos, para el presente estudio se estableció trabajar solo con tres indicadores: aerobios mesófilos de categoría 3, E. coli de categoría 6 y Salmonella sp. de categoría 10 a fin de establecer no solo el tiempo de vida útil; sino también, la inocuidad de su consumo. Siendo los límites inferior y superior los mostrados en la Tabla 1. En lo que respecta a los recuentos de las dos especies patógenas, estos siempre fueron "cero"; por lo tanto, el producto sangrecita de pollo es inocuo para el consumidor hasta el día 20 de su producción manteniéndolo en refrigeración.

Para establecer su tiempo de vida útil, es el recuento de aerobios mesófilos el de gran relevancia ya que las especies que conforman este grupo de microorganismos incluyen microorganismos descomponedores o alteradores del alimento (Perlera, 2015). La cantidad de AMV que se puede encontrar en un alimento proviene tanto de la materia prima utilizada como del proceso mismo de fabricación.

La sangre de pollo debido a su riqueza de nutrientes es fácilmente contaminada durante el proceso de su extracción por microorganismos del ambiente. Las verduras empleadas en la formulación, también contribuyen con su propia flora microbiana por lo que se esperaría recuentos 
altos de AMV. Otros elementos que influyen en el número de estos microorganismos, pero de manera negativa (disminuyéndolos) son las especias utilizadas para mejorar el sabor y por ende su aceptabilidad, pero que poseen actividad antimicrobiana y el efecto del procesado térmico al que fueron sometidos los ingredientes durante su preparación (cocción).

Mahecha y col. (2011), señalan que las especias consideradas con actividad antimicrobiana en orden decreciente son: orégano, cilantro, canela, tomillo, romero, perejil, mostaza y salvia; y que por su actividad pueden emplearse como alternativa al uso de conservantes químicos. Todos estos factores serían los que establecieron que el primer día de producción se tuvieran recuentos del orden de $10^{2}$ a $10^{3} \mathrm{UFC} / \mathrm{g}$ de AMV, por debajo de los límites, por lo que se estableció volver a evaluar el producto al día 15 y 20 de su conservación en refrigeración. Al día 15 los recuentos se elevaron a un orden de $10^{4} \mathrm{UFC} / \mathrm{g}$, permaneciendo aún dentro del rango de aceptabilidad y al día 20 si bien se superó el límite inferior, no así con el límite superior por lo que aún era apto para su consumo; a diferencia de los productos utilizados como contraprueba que tienen indicado en sus etiquetas como tiempo de apto para consumo 15 días a partir de su fecha de producción.

De los tres conservantes probados, el acetato más diacetato fue el que mantuvo recuentos menores, pero en ninguno de los casos se superó lo establecido como límite inferior $\left(5 \times 10^{4} \mathrm{UFC} / \mathrm{g}\right)$ hasta el día 15 de su conservación por lo que se determinó continuar con el análisis al día 20 donde los recuentos si bien superaban el límite inferior, se mantuvieron por debajo del límite superior $\left(5 \times 10^{5} \mathrm{UFC} / \mathrm{g}\right)$ siendo aún aptos para su consumo. Estos conservantes actúan difundiendo al interior de las células microbianas y causando un descenso del $\mathrm{pH}$ interno, lo que conduce a su vez a la inhibición de la respiración, fermentación/glicólisis y transporte activo; por lo cual, las células microbianas no pueden generar el ATP necesario para su crecimiento y reproducción, pero su efecto es reversible conforme aumenta la humedad del ambiente que rodea a los microorganismos.

Sensorialmente, con la primera evaluación se determinó que las características de textura y olor (Sig. < 0.05) varían en los cinco productos probados, mientras que el sabor y el color son similares entre sí (Sig. > 0.05). En la evaluación general, el mejor producto fue el preparado con Ácido cítrico + Sorbato de potasio que obtuvo una calificación promedio de 4.1 (Tabla 9), seguido del producto con Sorbato de potasio, acetatos, Redondos y Wong. En la evaluación global, se refleja que la percepción de los jueces le adjudicó el mayor valor al preparado con Ácido cítrico más Sorbato de potasio con un promedio de 4.1 (Figura 1), en segundo lugar, el de acetatos y diacetatos, en tercer lugar, el de Sorbato de potasio y en último lugar las marcas comerciales (Redondos y Wong).

En la segunda evaluación sensorial se determinó que la característica de color (Sig. < 0.05) varía en los tres productos con diferente conservante, mientras que el sabor, textura y olor son similares entre sí (Sig. > 0.05). La evaluación general refleja que la percepción de los jueces le adjudicó el mayor valor al preparado que contenía como conservante Ácido cítrico más Sorbato de potasio con una calificación promedio de 3.9 (Tabla 10 y Figura 
2), seguido de los productos con Sorbato de potasio y acetatos.

\section{Conclusiones}

- La sangrecita de pollo tiene proteínas de alto valor nutritivo, que, junto con el zinc y el hierro combaten la anemia, ayudan al buen desarrollo celular y brindan al cuerpo defensas contra distintas enfermedades. Contiene hierro hemínico y por ello, no necesita vitamina $\mathrm{C}$ o cítricos para una mejor absorción. Además, es un alimento que casi no aporta grasa al cuerpo (65 calorías), considerándose un alimento recomendable para las personas con problema de colesterol.
- En base a la investigación de evaluación sensorial, de acuerdo con la percepción de los jueces, se concluye que el producto "Sangrecita de pollo" con la mezcla de conservante Ácido cítrico más Sorbato de potasio obtiene una mejor aceptación.

- Microbiológicamente el producto sangrecita de pollo (bajos las tres presentaciones con las sustancias preservantes) es inocuo para el consumidor ya que cumple con los parámetros establecidos: no contiene los patógenos E. coli y Salmonella sp y su tiempo de vida útil, conservado en refrigeración, es de 20 días desde la fecha de producción.

\section{Referencias}

Balta, W. (2007). Química de los alimentos (5ta edición). Zaragosa: Acribia. www.analizacalidad.com

Bueno, V. 2015. Elaboración, calidad nutritiva de un bollo dulce relleno con sangre de pollo y su aceptabilidad en preescolares. Tesis Para optar el título profesional de licenciada en Nutrición. UNMSM, Facultad de Medicina, EAP. De Nutrición. Lima, Perú.

Galarza, R.R.O. (2012). Calidad nutricional de un producto extruido fortificado con dos niveles de hierro proveniente de harina de sangre bovina. Rev. de Invest. UNMSM: Anales de la Facultad de Medicina.73: S58.

Harris D. (2015). Quantitative Chemical Analysis. (9ena Edición). España, Reverté.
ISN. (2015). Anemia en la población infantil del Perú: aspectos clave para su afronte. Visto en: https:// docplayer.es/16574055-Anemiaen-la-poblacion-infantil-del-peru-aspectos-clave-para-su-afronte. html

Mahecha, H.S.; D. A. Restrepo y L. A. Carrasquilla. 2011. Influencia de Especias Naturales en la Vida Útil y Aceptación Sensorial de Salchicha Bratwurst. Rev.Fac.Nal.Agr.Medellín 64(1): 6007-6013.

Ministerio de Salud, Dirección General de Salud Ambiental (MINSA). 2008. Norma sanitaria que establece los criterios Microbiológicos de calidad sanitaria e inocuidad para los alimentos y bebidas de consumo humano. NTS No 071. 
EVALUACIÓN DEL USO DE CONSERVANTES: SORBATO DE POTASIO, ACETATOS Y DIACETATOS Y MEZCLA DE SORBATO DE POTASIO Y ÁCIDO CÍTRICO EN LA VIDA ÚTIL DEL PRODUCTO COMERCIAL “SANGRECITA DE POLLO”

OPS, OMS. (2002). "Condición de Salud de las Américas". Publicación científica $\mathrm{N}^{\circ}$ 524, ed. $2002 \mathrm{OPS}_{-}$ OMS, Vol. I. Pag. 34.

Perlera de Escalante, A. (2015). Evaluación de vida útil en productos cárnicos crudos, empacados al vacío y en bandeja termo formada, de la planta de cárnicos, Facultad Multidisciplinaria de Ilobasco, Universidad Católica de El Salvador. Anuario de Investigación. 419-426. Vol. 4 UNICAES.

Rengifo, J.M.; Ramírez, L. M, Restrepo, D.A., M. Cortes y Suarez, H.( 2010). Producción y propiedades funcionales de plasma bovino hidratado en embutido tipo salchi- chón. RCCP (revista colombiana de ciencias pecuarias). 23(2): 199206

Ruiz, M.M. (2009). "Evaluación de la morcilla castellana utilizando dos tipos de tripas comestibles (natural y colágeno)". Tesis de grado Previa la obtención del título de: Ingeniero zootecnista. Escuela superior politécnica de Chimborazo. Facultad de ciencias pecuarias. Riobamba, Ecuador.

Zagaceta. (2006). Costo y efectividad de la ingesta de sangre de pollo en el tratamiento de la anemia ferropénica en estudiantes de la E.A.P. de obstetricia de la Facultad de Medicina de la UNMSM - 2004. 
